\title{
Design of Bandpass Filter with High Selectivity Using Stepped Impedance Resonator
}

\author{
Xue-Liang Min, Hou Zhang, Tao Zhong, Qiang Chen \\ Air Force Engineering University, Xi'an, Shanxi Province, China, 710051 \\ E-mail: MinXL08@163.com
}

\begin{abstract}
In this paper, a novel bandpass filter (BPF) with high selectivity and great out-of band suppression is proposed by using stepped impedance resonators (SIR) and defected ground structure. The measured 3-dB bandwidth of the proposed $\mathrm{BPF}$ is $2.62-2.72 \mathrm{GHz}$; the maximum simulated insertion loss is $0.4 \mathrm{~dB}$ within passband. Besides, there are four transmission zeros located at 2.12, 2.54, 2.84, and $3.80 \mathrm{GHz}$, the out-of band suppression is better than 20 $\mathrm{dB}$, indicating that the proposed BPF has great suppression. Moreover, the measured and simulated filter characteristics are in agreement, which means that this design is effective. The characteristic of high selectivity is meaningful in wireless communication.
\end{abstract}

Keywords-high selectivity; stepped impedance resonator; bandpass filter; wireless communication

\section{INTRODUCTION}

Modern development in wireless communication systems has induced a increasing demand for planar microstrip microwave device. Specially, microstrip filter is a major component in communication systems. As various application requirements occur, great deals of filters with different characteristics are proposed [1]-[9]. In [1], a compact ultrawideband (UWB) bandpass filter (BPF) with wide notched-band is proposed; the structure is composed of a U-shaped open-circuited line. In [2], a compact filter using defected stepped impedance resonator (DSIR) is presented. The resonator property of DSIR is studied and compared with microstrip SIR. Besides, stepped-impedance resonators are utilized to realize the dual-band bandstop filter [3], and a meandered-line stepped-impedance resonator [4] is used in the design of dual-band bandstop filter. In [5], a bandpass filter with two transmission poles in passband using a single ring resonator is designed. In [6], simple designs of dual bandpass and bandstop filters using microstrip open-loop resonator are presented. It uses a simple technique avoiding the method of extracting coupling coefficient matrices to realize the filter. In addition, a compact UWB bandpass filter with tunable notch band based on folded SIR is fabricated in [7]. Transversal signal-interaction with three paths [8] and meandered slot defected microstrip structure [9] are also used in the design of dual-band bandstop filter. In recent years, many novel filters [10]-[12] are fabricated adopting different structure. Stepped impedance resonator and defected ground structure are usually adopted in these novel designs [13]-[18].
In this letter, a bandpass filter with high selectivity is proposed, to get better out-of band suppression and four transmission zeros, stepped impedance resonators (SIR) and defected ground structure are adopted. The 3-dB bandwidth of the proposed BPF is $2.62-2.72 \mathrm{GHz}$. The result of Sparameter has four transmission zeros, the creation is due to the cross coupling in stepped impedance resonators and mode of feeding.

\section{STRUCTURE OF BANDPASS FILTER}

The geometries of stepped impedance resonator is shown in Fig. 1. The size of the structure are $11=5.3 \mathrm{~mm}, 12=9.8 \mathrm{~mm}$, $\mathrm{w} 1=2.5 \mathrm{~mm}, \mathrm{w} 2=2 \mathrm{~mm}$.

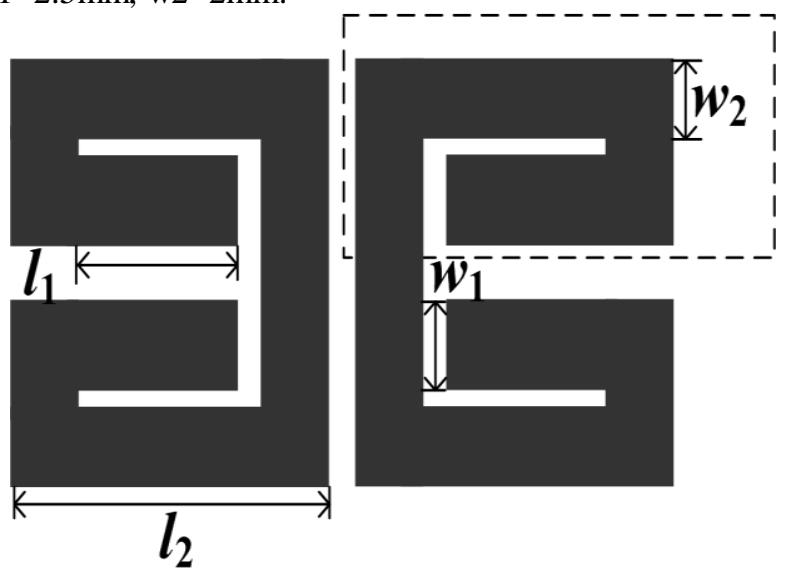

Figure 1. The structure of stepped impedance resonators.

Proposed resonator is consisted of two steppedimpedance resonators. As shown in Fig. 2, to obtain the input impedance (Zin), the SIR is partitioned into two sections: the first section has characteristic impedance (Z1) and electrical length ( $\theta 1)$; the second section consists of stubs with characteristic impedance (Z2) and electrical length $(\theta 2)$. It is apparent to evaluate the overall input impedance by cascading the ABCD matrix of section 1 and 2 . The ABCD matrix of section 1 is deduced as (1)

$$
\left[\begin{array}{ll}
A & B \\
C & D
\end{array}\right]_{1}=\left[\begin{array}{cc}
\cos \theta_{1} & \mathrm{j} Z_{1} \sin \theta_{1} \\
j \frac{1}{Z_{1}} \sin \theta_{1} & \cos \theta_{1}
\end{array}\right]
$$

The ABCD matrix of section 2 is deduced as (2) 


$$
\left[\begin{array}{ll}
A & B \\
C & D
\end{array}\right]_{2}=\left[\begin{array}{cc}
\cos \theta_{2} & \mathrm{j} Z_{2} \sin \theta_{2} \\
j \frac{1}{Z_{2}} \sin \theta_{2} & \cos \theta_{2}
\end{array}\right]
$$

After cascading section 1 and 2, the ABCD parameters of SIR could be got. The overall input impedance (Zin) of the SIR is deduced as (3)

$$
Z_{\text {in }}=\frac{R_{\mathrm{Z}}\left(1-\tan ^{2} \theta_{1}\right)\left(1-\tan ^{2} \theta_{2}\right)-2\left(1+R_{z}^{2}\right) \tan \theta_{1} \tan \theta_{2}}{j \frac{1}{Z_{2}}\left(R_{z}-\tan \theta_{1} \tan \theta_{2}\right)\left(R_{z} \tan \theta_{1}+\tan \theta_{2}\right)}
$$

Yin=1/Zin, at the resonance condition, Yin= 0 such that

$$
R_{\mathrm{Z}}=Z_{2} / Z_{1}=\tan \theta_{1} \tan \theta_{2}
$$

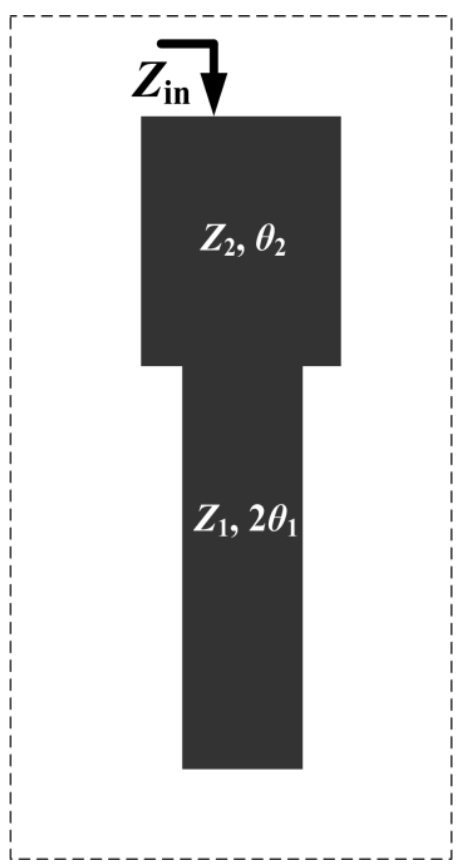

Figure 2. The general SIR.

The schematic of the proposed bandpass filter is demonstrated in Fig. 3. In this design, two stepped impedance resonators are symmetrically-arranged. The substrate has a relative dielectric constant of 2.2 and the thickness of $0.508 \mathrm{~mm}$, at the bottom side of the substrate, there is defected ground structure. A ring and a smaller ring form a unit; defected ground structures have six units totally. At the top side of the substrate, it is the structure of stepped impedance resonators. The distance between two stepped impedance resonators is chosen as $\mathrm{d} 2=0.8 \mathrm{~mm}$ which results in a external coupling. These stepped impedance resonators have the same dimensions and they are symmetricallyarranged. Other parameters are $\mathrm{d} 1=0.4 \mathrm{~mm}, 13=18.4 \mathrm{~mm}$, $14=14.1 \mathrm{~mm}, 15=6 \mathrm{~mm}, 16=7.9 \mathrm{~mm}, \mathrm{w} 3=3 \mathrm{~mm}$.

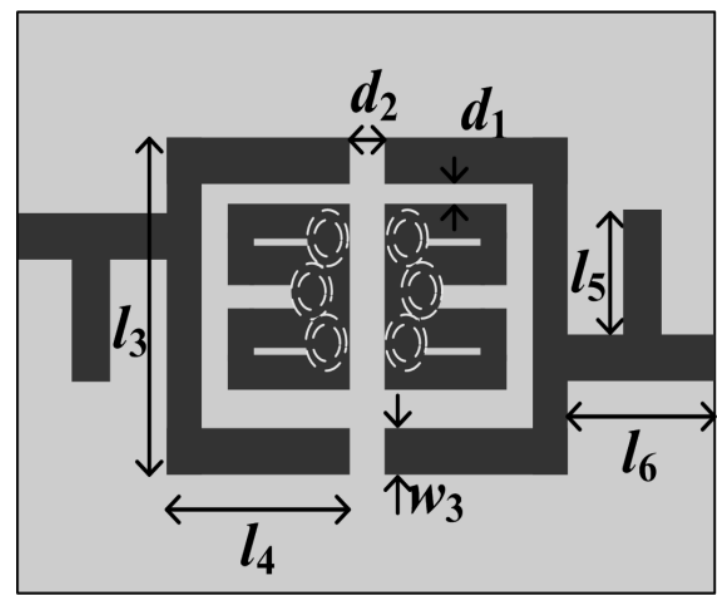

Figure 3. The schematic of bandpass filter.

\section{SIMULATION AND MEASUREMENT}

Based on the proposed structure, the results of Sparameter are obtained by using High Frequency Structure Simulator (HFSS) software as shown in Fig. 4. As can be observed, the result curve of S-parameter has four transmission zeros and the filter has great out-of band suppression. The characteristic of passband is also great.

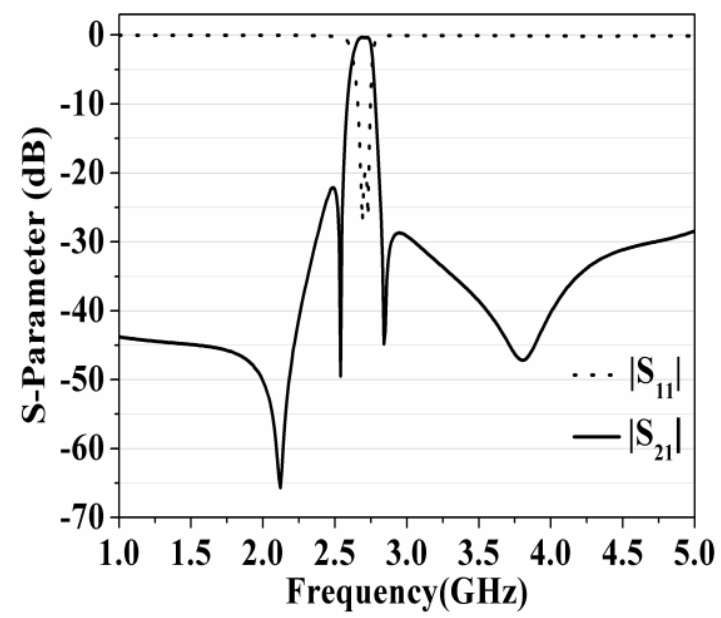

Figure 4. Simulated results.

By adjusting the size of structure, the filtering effects of structures are different. Fig. 5 demonstrates the S-parameter variations according to the value of 11 . In this case, 11 varies from 4.3 to $5.3 \mathrm{~mm}$, while the values of other parameters are fixed. Results display that the characteristics of $3-\mathrm{dB}$ bandwidth and out-of band suppression are affected by value of 11 . When 11 varies from $4.3 \mathrm{~mm}$ to $5.3 \mathrm{~mm}$, the selectivity of filter is getting greater.

From the above mentioned, we could know that the dimensions of structure are critical for the selectivity of the proposed filter. Changing the value of 11 , the working frequency band is affected. 
Fig. 6 exhibits comparison of the S-parameter via changing the value of $\mathrm{d} 2$. Simulated results indicate that the out-of band suppression is being better when $\mathrm{d} 2$ varies from $0.6 \mathrm{~mm}$ to $0.8 \mathrm{~mm}$, being worse when $\mathrm{d} 2$ varies from $0.8 \mathrm{~mm}$ to $1.0 \mathrm{~mm}$. It also displays that the characteristics of $3-\mathrm{dB}$ bandwidth and out-of band suppression are affected by value of $\mathrm{d} 2$. When $\mathrm{d} 2$ is $0.8 \mathrm{~mm}$, the performance of structure is great.

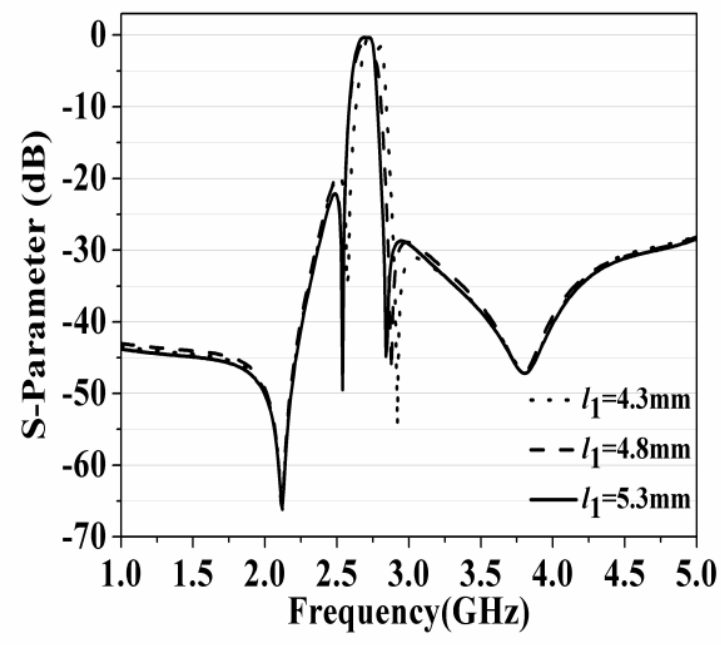

Figure 5. S-parameter variations according to the 11.

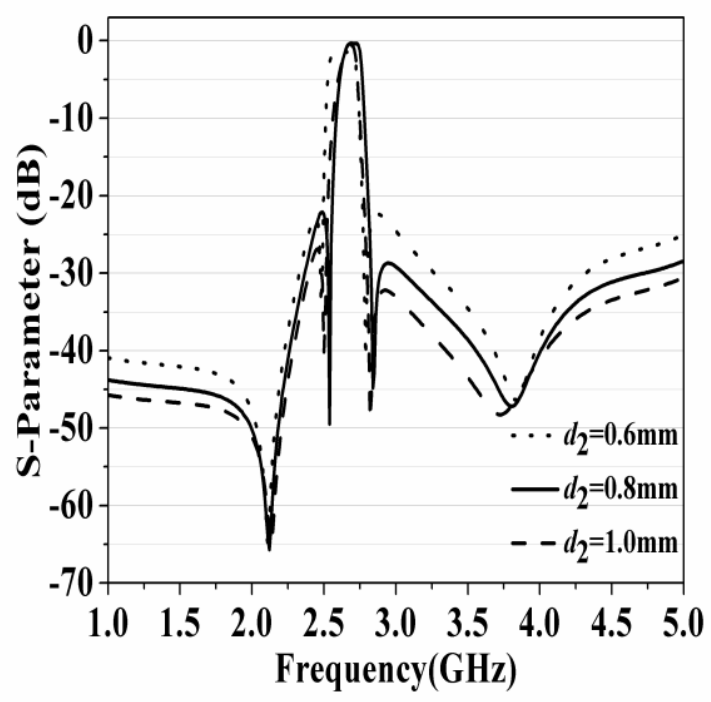

Figure 6. S-parameter variations according to the d2.

Fig. 7 plots the simulated and measured S-parameter curves against frequency. The bandpass filter has four transmission zeros located at 2.12, $2.54,2.84$, and $3.80 \mathrm{GHz}$ and the insertion loss within passband is getting worse. In totally, the simulated results agree with measurements.

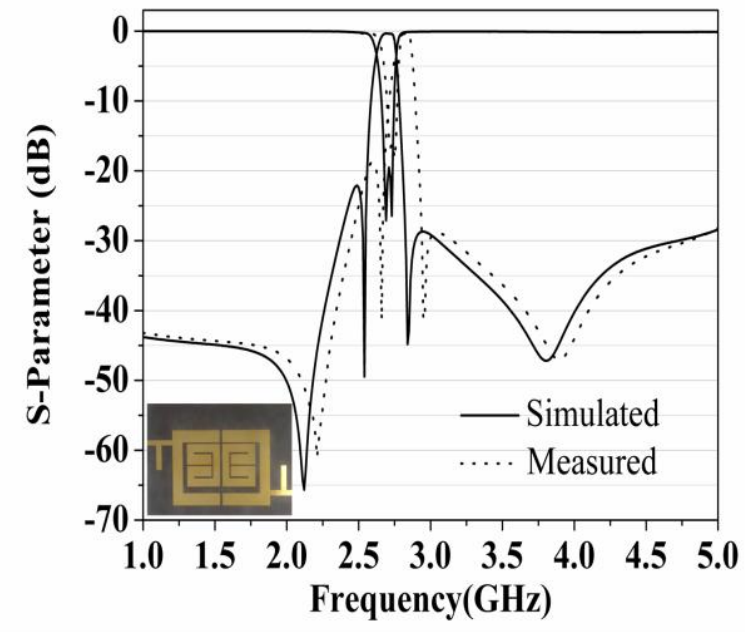

Figure 7. Simulated and measured results.

\section{CONCLUSION}

A bandpass filter (BPF) with high selectivity and four transmission zeros is proposed. Low insertion loss and great out-of band suppression are introduced by the defected ground structure. Stepped impedance resonators are symmetrically-arranged, defected ground structure consists of six rings units. Owing to the cross coupling in stepped impedance resonators and mode of feeding, four transmission zeros are induced. Results indicate that the proposed filter has great selectivity; it can be used in wireless communication.

\section{REFERENCES}

[1] Seyyed Jamal Borhani, M. Amin Honarvar, Bal.S.Virdee,"High Selectivity UWB Bandpass Filter with a Wide Notched-Band," Microwave And Optical Technology Letters, vol.57, No.3, March 2015

[2] Bian Wu, Chang-hong Liang, Pei-yuan Qin, QiLi,"Compact DualBand Filter Using Defected Stepped Impedance Resonator," IEEE Microwave And Wireless Components Letters, vol.18, No.10, October 2008.

[3] Rajendra Dhakal, Nam-Young Kim,"A Compact Dual-band Bandstop Filter Using A Circular, Folded, Symmetric, MeanderedLine,Stepped-Impedance Resonator," Microwave And Optical Technology Letters, Vol. 56, No. 10, October 2014.

[4] Cheng Zhang, Jun-Ping Geng, Rong-Hong Jin, Xian-Ling Liang, Liang Liu"Dual-Wideband Bandstop Filter Using Stepped Impedance Coupled-Lines," Microwave And Optical Technology Letters, Vol. 57, No. 10, October 2015.

[5] Sha Luo, Lei Zhu,"A Novel Dual-Mode Dual-BandBandpass Filter Based on a Single Ring Resonator," IEEE Microwave And Wireless Components Letters, vol.19, No.8, August 2009.

[6] Subash Vegesna, Mohammad Saed," Microstrip Dual-Band Bandpass And Bandstop Filters," Microwave And Optical Technology Letters, vol. 54, No.1, January 2012.

[7] F. Wei, L. Chen, X.-W. Shi, B. Liu,"Compact UWB bandpass filter with tunable notch band based on folded SIR," Electronics Letters, Vol.47, No.22, October 2011. 
[8] Qing-Xin Chu, Lei-lei Qiu," Sharp-Rejection Dual-Band Bandstop Filter Based On Signal Interaction With Three Paths," Microwave And Optical Technology Letters, Vol. 57, No. 3, pp.657-660, 2015.

[9] H. Ning, J. Wang, Q.Xiong, L. Mao," Design Of Planar Dual And Triple Narrow-Band Bandstop Filters With Independently Controlled Stopbands And Improved Spurious Response," Progress In Electromagnetics Research,Vol.131, pp.259-274, 2012.

[10] Wenquan Che, Wenjie Feng, Kuan Deng," Microstrip Dual-Band Bandstop Filter Of Defected Ground Structure and Stepped Impedance Resonators," International Journal of Electronics, Vol.97, No.11, pp.1351-1359, 2010.

[11] X.H. Jin, X.D. Huang, and C.H. Cheng, "Bandstop response of microstrip stepped impedance rings with rotational symmetry, "Electron Lett, Vol.49, pp.121-123, 2013.

[12] Tao Pan, Kaijun Song, Yong Fan,"Compact Dual-bandstop Filter Based On Composite Right/left Handed Transmission Line," Microwave And Optical Technology Letters, Vol. 55, No. 5, May 2013.

[13] Qing-Xin Chu, Fu-Chang Chen,"A Compact Dual-Band Bandpass Filter Using Meandering Stepped Impedance Resonators," IEEE
Microwave And Wireless Components Letters, vol.18, No.5, May 2008.

[14] R. Dhakal and N.Y. Kim, "A compact symmetric microstrip filter based on a rectangular meandered-line stepped impedance resonator with a triple-band bandstop response, " Sci World J 2013 (2013).

[15] Gyan Raj Koirala, Bhanu Shrestha, Nam-Young Kim,"Compact dualwideband bandstop filter using a stub-enclosedstepped-impedance resonator ," International Journal of Electronics and Communications (AEÜ), vol. 70, pp. 198-203, 2016.

[16] Mostafa Mashhadi, Nader Komjani,"Design of Dual-Band Bandpass Filter With Improved Upper Stopband Using Novel SteppedImpedance Resonator,"Microwave And Optical Technology Letters, vol.56, pp. 603-606, 2014.

[17] Kuo-Sheng Chin, Jun-Hong Yeh, Shuh-Han Chao,"Compact DualBand Bandstop FiltersUsing Stepped-Impedance Resonators," IEEE Microwave and Wireless Components Letters,,vol. 17, pp. 849-851, 2007.

[18] Pankaj Sarkar, Manimala Pal, Rowdra Ghatak,"A Compact Dual Stopband Bandstop Filter Using Defected SIR and Hilbert Shape Fractal Structure," Microwave And Optical Technology Letters, vol.58,pp.1345-1347,2016. 